\title{
A ENFERMAGEM COMUNITÁRIA NO CURRICULO DO CURSO DE GRADUAÇÃO EM ENFERMAGEM (PARTE II) *
}

\author{
Maria Jacyra de Campos Nogueira**
}

NOGUEIRA, M. J. de C. A enfermagem comunitária no currículo do curso de graduação em enfermagem (parte II). Rev. Esc. Enf. USP, São Paulo, 13(2): 171-181, 1979.

A primeira parte deste artigo tratou de conceitos básicos sobre saúde, saúde pública $e$ enfermagem comunitária. Esta segunda parte contém a problemática do ensino de enfermagem comunitária, em nosso meio, bem como as sugestões para a inclusão de aspectos preventivos e comunitários no currículo de enfermagem.

\section{ENSINO DE ENFERMAGEM E DE ENFERMAGEM COMUNITÁRIA - PROBLEMÁTICA EM NOSSO MEIO}

Observando as atividades de enfermagem em alguns hospitais onde tivemos a oportunidade de trabalhar ou mesmo de visitar, verificamos que em alguns deles, vários aspectos importantes da assistência de enfermagem eram relegados a um segundo plano ou, até mesmo, esquecidos ${ }^{5}$.

0 que mais nos chamou a atenção foram as atividades relacionadas ao ensino ou orientação do paciente, quer nas unidades de internação, quer nos ambulatórios, tais como orientações simples sobre a doença de que estavam acometidos, os exames que teriam de fazer, o preparo para a alta e posterior continuidade dos cuidados no domicílio e as medidas para evitarem a reinternação.

A maioria das enfermeiras concordavam que essas atividades eram importantes mas que não havia possibilidade de serem executadas, por dois motivos: a falta de tempo disponível, resultante do número insuficiente de pessoal de enfermagem e a inexistência de pessoal preparado para tal. Com relação ao segundo motivo, apontavam as atividades de ensino como especificas da enfermagem de saúde pública e, pelo fato do hospital não poder contar com esse profissional, aquelas atividades não eram executadas.

Em outros hospitais, encontramos situação diversa: as atividades educativas eram incluídas no cuidado aos pacientes internados ou nos ambulatórios. Contudo, as atividades educativas como palestras, reuniões de grupo, etc., eram denominadas de" atividades de enfermagem de saúde pública" e sua execução estava a cargo de enfermeiras com ou sem especialização em saúde pública.

Em outros hospitais, ainda, encontramos serviços organizados denominados de "serviço de enfermagem de saúde pública". Localizados geralmente em ambulatórios, as suas atividades, resumidamente, eram: preparo e orientação de pacientes após a consulta médica, distribuição de medicamentos gratuítos, orientação

\footnotetext{
* Trabalho apresentado no I Encontro Nacional de Educação em Enfermagem, São Paulo, 1979.

** Professor Assistente Doutor das disciplinas Enfermagem Preventiva e Comunitária e Saúde da Comunidade da EEUSP.
} 
quanto à prescrição médica, planejamento e execução de cursos para grupos de pacientes internados ou não, orientação de um membro da família para o retorno do paciente ao lar ,visitação domiciliária para prestação de cuidado a um doente no lar, aplicação de vacinas e de testes de imunidade. Tais atividades, embora também denominadas de "enfermagem de saúde pública", eram executadas por enfermeiras que geralmente haviam feito curso de saúde pública.

Essas observações, na época, levaram-nos a crer que as enfermeiras identificavam as atividades educativas como sendo especificas de enfermagem de saúde pública e não como responsabilidade de qualquer enfermeira; e que, muitas vezes, essas atividades não eram executadas por insegurança e falta de preparo da enfermeira hospitalar.

Devido a esses problemas, em 1972 realizamos uma pesquisa no Estado de São Paulo ${ }^{5}$, com os seguintes objetivos:

- levantar informações sobre como estava sendo realizado o ensino de enfermagem de Saúde Pública no Estado de São Paulo, nas seis escolas de enfermagem existentes na época;

- verificar as opinióes a respeito do que as enfermeiras formadas por essas escolas, entre 1950 e 1970, consideravam como "conhecimento de enfermagem de saúde pública" adquirido no curso de graduação que haviam realizado.

$\mathrm{Na}$ época, os dados levantados nos deram ,entre outras, as informações abaixo referidas.

Mais de metade das enfermeiras (62\%) indicou incluir "aspectos de enfermagem de saúde pública" no cuidado dispensado aos seus pacientes. Esses aspectos ou atividades diziam respeito, em primeiro lugar $(63 \%)$, aos que reunimos sob a denominação de "atividades educativas"; indicadas pelas enfermeiras como sendo as únicas atividades de enfermagem de saúde pública executadas por elas, referiam-se, em geral, ao ensino de pacientes e familiares, por meio de entrevistas ou palestras;

Em segundo lugar (10\%), apareceram os cuidados com doenças transmissíveis, com as seguintes atividades: pesquisa de focos e isolamento no hospital e no domicilio, no caso de doenças infecto-contagiosas; prevenção de infecções no meio hospitalar (cuidados com roupas dos doentes, com material de injeções, com o lixo e com o ambiente físico de um modo geral);

Em terceiro lugar ( $6 \%$ ) citam o controle de gestantes e crianças sadias, compreendendo a consulta de enfermagem ou o atendimento pré e pós consulta médica e as visitas domiciliárias, com a finalidade de prestar cuidados no domicílio ou simplesmente orientar a família, principalmente nas áreas da saúde materno-infantil e das doenças transmissíveis. Em quarto e quinto lugares, aplicação de vacinas e de testes de imunidade, principalmente o de Mantoux (5\%) e os entrosamento com os recursos da comunidade tais como: centros de saúde, outros hospitais ou outras instituições e encaminhamento dos pacientes para as mesmas (3\%).

Por fim, foram mencionadas algumas atividades que não chegaram a especificar, detalhadamente, e que denominaram de "assistênica social" $(2 \%)$ ou de "cuidado integral" (1\%), aparecendo, ainda, em último lugar $(1 \%)$, o preparo dos pacientes de ambulatório para a consulta médica. 
Os motivos apontados pelas enfermeiras, visando justificar porque elas incluiam os denominados "aspectos de enfermagem de saúde pública" no cuidado, estavam, em primeiro lugar, ligados à execução de atividades educativas, isto é, a maior porcentagem $(38 \%)$ dizia inclui-los, "porque havia orientação do paciente e sua família". Em segundo lugar $(23 \%)$, as justificativas eram ligadas a porblemas econômico-sociais dos pacientes, tais como: "eram muito pobres", "não tinham instrução suficiente", "tinham tabús relativos à saúde", "não sabiam utilizar os recursos que dispunham". Em terceiro lugar (15\%), as justificativas eram ligadas às condições que obrigavam ou favoreciam a inclusão daquelas atividades, isto é, "as finalidades da instituição" ou "do serviço de enfermagem de saúde pública do hospital". Finalmente, justificativas como: "devem fazer parte do cuidado" ( $9 \%)$, "são importantes" $(5 \%)$ ou outras $(1 \%)$.

Com relação à opinião das enfermeiras sobre quais seriam as atividades de enfermagem de saúde pública no hospital, apareceram, em primeiro lugar (55\%), as atividades relacionadas ao ensino de pacientes e familiares, sendo que, para algumas enfermeiras, foram as únicas apontadas. As visitas domiciliárias apareceram em segundo lugar $(12 \%)$, tendo como objetivo "dar continuidade ao cuidado de enfermagem no domicílio". Outras atividades, a seguir, foram: a vacinação $(8 \%)$, "várias" $(6 \%)$, "cuidados com as doenças transmissíveis" $(5 \%)$, "entrosamento com recursos da comunidade" (4\%), "coleta de dados do ambiente social do paciente" $(3 \%)$, "assistência social" $(2 \%)$, "todas de enfermagem de saúde pública" $(2 \%)$, além de algumas cujo significado não conseguimos decifrar $(3 \%)$.

Da análise desses dados conclui-se que, na época embora as enfermeiras estivessem preocupadas em tornar a assistência de enfermagem mais efetiva, nenhuma soube conceituar o que seria uma atividade de enfermagem de saúde pública, confundindo-a com a assistência educativa e com as ações preventivas que qualquer enfermeira deve executar, independente de ser ou não especializada, ou de existir, nos hospitais, serviço organizado denominado de "enfermagem de saúde pública".

Quanto ao ensino de enfermagem de saúde pública, verifica-se que, em nosso meio, ele é bem antigo. Desde 1923 que as escolas de enfermagem do País ${ }^{2}$ vêm se preocupando em incluir, nos currículos dos cursos de graduação em enfermagem, o ensino de enfermagem de saúde pública; entretanto, os cursos específicos de enfermagem de saúde pública são bem mais recentes ${ }^{3}$.

De acordo com os dados da pesquisa realizada em $1972^{5}$, verificou-se o seguinte:

- com relação aos objetivos do currículo, todas as escolas de enfermagem do Estado de São Paulo, na época, informaram que estes visavam formar profissionais capazes de prestar cuidados não só a doentes, mas também a sadios, à família e à comunidade, embora somente três, das seis escolas existentes, ministrassem no último ano, um curso de habilitação em saúde pública. Entre essas seis escolas, ainda, quatro informaram preparar seus alunos para a chefia de unidades de enfermagem ou de serviços de enfermagem, não só em hospitais mas, também, em centros de saúde;

- com relação ao ensino da assistência de enfermagem de saúde pública, as seis escolas afirmaram sua existência no curso de graduação em enfermagem; 
somente duas ,entretanto, justificaram esse ensino, uma dizendo que "o estudante devia ter visão global dos aspectos preventivos e curativos" e outra, que esse ensino "visava atender aos objetivos da escola, quanto ao tipo de profissional que se desejava formar".

Foi verificado, tendo como base os conceitos de HEIDGERKEN ${ }^{4}$ e de RIVERA 7, que esse ensino era realizado de três formas: 1. isoladamente, quando não estava vinculado a nenhuma outra disciplina, fazendo parte somente do conteúdo das disciplinas denominadas "de saúde pública"; 2 . de forma correlacionada, quando os mesmos eram ensinados concomitantemente a outras disciplinas, embora fossem sempre os docentes responsáveis pelas disciplinas de enfermagem de saúde pública que os ministrassem; 3 . de forma integrada, quando os docentes de outras disciplinas aplicavam os conhecimentos de saúde pública, ou os docentes de enfermagem de saúde pública participavam no ensino de outras disciplinas.

Nas seis escolas de enfermagem encontrou-se o seguinte:

— em três delas, o ensino de "enfermagem de saúde pública" era ministrado tanto sob a forma correlacionada, como isolada; na quarta, era correlacionado "a quase totalidade das disciplinas" dos curriculo, quer de enfermagem ou não, na quinta, a correlação era realizada somente com a disciplina Fundamentos de Enfermagem, sendo que os responsáveis pelas outras disciplinas de enfermagem estavam tentando integrar os aspectos de saúde pública no conteúdo de sua disciplina; e finalmente, na sexta a correlação era feita com as disciplinas Enfermagem Médico-Cirúrgica e Materno-Infantil;

- em uma escola, o ensino era realizado das três formas já mencionadas, sendo que a correlação era feita com a disciplina Enfermagem Materno-Infantil e a integração, por outras disciplinas tais como :Antropologia, Sociologia, Didática e Metodologia de Pesquisa;

- em uma escola, era correlacionado com as seguintes disciplinas: Fundamentos de Enfermagem, Enfermagem Médico-Cirúrgica e Enfermagem MaternoInfantil;

- em uma, finalmente, era dado de forma inteiramente isolada ou independente de outras disciplinas.

A maneira pela qual esses ccnhecimentos eram distribuidos no curriculo também não era uniforme, mas a maior parte era ministrada no último ano, havendo preocupação em lhe dar um cunho prático, sendo que todas as escolas informaram que propiciavam experiências em serviços de saúde da comunidade.

Quanto aos aspectos que foram denominados de "enfermagem de saúde pública" e que as escolas, na época, ministravam, referem-se ao seguinte: Saneamento (seis escolas), Estatística Vital (cinco), Epidemiologia (quatro), Nutrição em Saúde Pública (três), Estudo de Problemas Brasileiros (uma), Tisiologia em Saúde Pública (uma) e Saúde da Comunidade (uma).

Com relação aos aspectos de "enfermagem de saúde pública" que os docentes das escolas de enfermagem consideravam como devendo ser incluídos no curriculo de graduação em enfermagem, todas as escolas referiram-se à "Educação sa- 
nitária", sendo que uma escolı mencionou somente esse, como necessário. Em seguida, apareceram os conhecimentos relacionados à "higiene materno-infantil" (três escolas), ao saneamento e à epidemiologia (três), à administração sanitária (duas) e, em menor número, os conhecimentos relativos às "doenças transmissíveis", "higiene do escolar", "higiene do adulto", "cuidados com sadios", "higiene industrial", "prevenção de doenças", "saúde da comunidade", "higiene do trabalho", "trabalho em zona rural". $\mathrm{O}$ assunto "serviços a familias" foi mencionado somente uma vez.

Na ocasião da pesquisa, segundo as informações obtidas, malgrado a preocupação das escolas, nada era feito para ensinar aos alunos a prestação de uma assistência mais integral ao paciente, visando aspectos de prevenção, educação sanitária, problemas prioritários de saúde do País, conhecimentos básicos de saúde pública necessários à formação de qualquer membro da equipe de saúde pública, ou de qualquer sanitarista, no que se refere a metodologia, funções específicas da enfermagem comunitária. Também a idéia do que ensinar como "enfermagem de saúde pública", era muito heterogênea nas diferentes escolas.

Se realizassemos um novo levantamento, sete anos após o que foi descrito, será que haveria mudanças? Será que realmente temos idéia, em nossos dias, do que ensinar ou como ensinar, em nossas escolas, sob o nome de enfermagem comunitária ou de saúde pública?

Com relação a esse ensino, os peritos da Organização Mundial de Saúde ${ }^{6}$ dizem o seguinte: o isolamento geográfico, as más comunicações, o meio ambiente desfavorável que expõe às enfermidades transmissiveis e à má nutrição, os serviços de saúde deficientes, a falta de saneamento e as escassas oportunidades em matéria de educação fizeram com que os serviços de saúde e a formação do pessoal de saúde se tenha centralizado no hospital. A enfermagem seguiu esta mesma tendência e a maior parte do ensino da enfermagem, senão toda, prepara a enfermeira para o trabalho nos hospitais, mais do que para o serviço na comunidade, que é onde há mais necessidade de assistência e proteção sanitária; os enfermos hospitalizados, aos quais é dedicada grande proporção dos serviços de enfermagem, representam uma proporção mínima da população necessitada de assistência.

As enfermeiras não estão sendo preparadas de acordo com as necessidades e recursos da população, pois as escolas de enfermagem centralizam sua atenção na teoria e na prática da assistência aos enfermos hospitalizados, dando pouca ou nenhuma atenção à promoção e proteção da saúde, bem como à reabilitação do indivíduo após a alta hospitalar. Nos cursos se descuida, inclusive, da assistência aos enfermos não internados em instituições, não sendo levado em consideração que, em qualquer comunidade, o seu número supera, em muito, ao número de enfermos hospitalizados.

Em São Paulo, mesmo a enfermeira que trabalha nos serviços de saúde pública e que teria mais oportunidades de prestar assistência de enfermagem comunitária mais efetiva, ainda não o conseguiu. Vários fatores parecem estar influenciando esse fato. $O$ primeiro é o total desconhecimento e a insegurança, por parte de algumas enfermeiras, das suas funções na equipe e da metodologia da assistência de enfermagem comunitária; o segundo é o desconhecimento dos outros profissionais a respeito do papel da enfermeira e do pessoal auxiliar de enfermagem nos serviços de saúde pública. 
Em decorrência verificam-se, nos centros de saúde de São Paulo, os seguintes problemas: a enfermeira, sem funções e atribuições definidas e conhecidas, é um elemento que se ocupa de muitas tarefas, menos a de prestar assistência de enfermagem comunitária, seja diretamente por ela própria, ou indiretamente, pelo pessoal auxiliar por ela supervisionado. É mais um funcionário administrativo, exercendo funções burocráticas que qualquer bom funcionário administrativo exerceria muito bem. A falsa idéia do que seja um verdadeiro trabalho em equipe faz com que a assistência de enfermagem exercida pelo pessoal auxiliar seja orientada e supervisionada por outros profissionais da equipe, como assistentes sociais, educadores sanitários ou de saúde pública e médicos; estes, por mais bem intencionados e competentes que sejam em suas respectivas funções específicas, não o são e nem podem ser nas funções específicas de enfermagem.

Em um centro de saúde de Capital de São Paulo, por exemplo as visitadoras prestam assistência centralizada no indivíduo e dicotomizada em relação aos aspectos integrais do ser humano. Essas visitadoras, em vez de generalistas ou polivalentes, em vez de abordarem a família como unidade de serviço da enfermagem, tornaram-se verdadeiras "especialistas" numa área de atendimento individual, chegando mesmo a ser denominadas visitadoras de "pediatria", de "saúde mental", etc. As visitas, por sua vez ,foram denominadas de "visitas de pediatria", "de saúde do adulto", "de saúde mental", "de puerpério", etc. Embora meritórias nas suas intenções, essas visitas não visavam a família, com problemas prioritários de saúde da criança, de saúde do adulto, de saúde materna ou de saúde mental, mas apenas serviam para verificar e orientar clientes faltosos às consultas médicas, orientar sobre tratamentos médicos específicos, etc. Esse tipo de serviço, além de estar completamente desvinculado da filosofia atual da assistência de enfermagem comunitária, é muito caro, pois, para uma mesma família com problemas prioritários de saúde materna, de saúde da criança e, por exemplo, concomitantemente, de saúde mental, terão que ser realizadas, no mínimo, três visitas, por três visitadoras diferentes. Esta forma não atende às necessidades e motivações reais da família e sim às necessidades e motivações que o serviço e o pessoal da equipe supõem serem as da família.

Com relação à metodologia do processo de enfermagem, uma pesquisa ${ }^{1}$ realizada em São Paulo, junto às enfermeiras da Secretaria de Estado da Saúde ,mostrou o seguinte: na função assistencial exercida pela enfermeira de saúde pública, somente uma pequena minoria delas $(14,30 \%)$, demonstrou conhecer a seqüência lógica das etapas básicas do processo de enfermagem; um número significativo $(71,40 \%)$ apresentou erros de conhecimento referentes à seqüência das etapas do referido processo; uma porcentagem também pequena $(14,30 \%)$ não respondeu à pergunta do formulário referente às etapas do processo, o que poderia sugerir, segundo o autor da pesquisa, desconhecimento do assunto.

A atuação da equipe de saúde, inclusive da enfermeira, encontrada nos serviços de prestação de assistência à comunidade acima descritos, além de desestimular novos profissionais a trabalharem nesses serviços, serve de modelo negativo para as escolas de enfermagem que estão procurando melhorar o ensino de enfermagem comunitária, inovando o conteúdo do seu ensino.

Quais seriam as modificações a serem feitas para sanar tais problemas? 
Segundo os peritos da Organização Mundial de Saúde ${ }^{6}$, fazem-se necessárias, entre outras, modificações na formação da enfermeira para a assistência à comunidade - não modificações de conteúdo técnico ou de métodos didáticos, mas da concepção do que seja enfermagem - e reformulação de currículos para atender às necessidades das populações de cada região.

As sugestões a seguir apresentadas não têm por finalidade esgotar o assunto nem transformar completamente o currículo mínimo vigente dos cursos de graduação em enfermagem ${ }^{3}$. Elas visam dar sugestões sobre como poderiam ser introduzidos no currículo aspectos de saúde comunitária, de prevenção e de enfermagem comunitária, que dessem maior segurança às enfermeiras para o trabalho em equipe, nos serviços de saúde pública, e para a prestação de assistência de enfermagem mais efetiva à população.

\section{SUGESTÕES PARA A INCLUSÃO DE ASPECTOS PREVENTIVOS E COMUNITÁRIOS NO CURRICULO MINIMO DOS CURSOS DE GRADUAÇÃO EM ENFERMAGEM}

\section{Conceitos básicos:}

- a saúde não é um estado ideal, mas um estado em que o indivíduo alcança um nível funcional ótimo, dentro da cultura, época e papel que desempenha na sociedade em que vive e dentro das suas limitações e capacidades individuais; a saúde é reconhecida como um dos direitos humanos básicos e a assistência sanitária é um meio importante de proteção a esse direito;

- a doença é um episódio na vida do indivíduo;

- a enfermagem visa o ser humano como um ser em sua tripla dimensão: física, psíquica e social, em interação com o meio ambiente;

- as ações de enfermagem, na prestação de assistência à saúde, desenvolvem-se abrangendo todos os níveis de prevenção dentro do contexto global e ecológico do ser humano e do fenômeno saúde-enfermidade;

- a enfermagem é exercida, atualmente, diretamente por enfermeiras (os) e, indiretamente, por pessoal auxiliar treinado e supervisionado por enfermeiras (os);

- a (o) enfermeira (o) tem as seguintes funções: a assistencial específica, que consiste em assistir ao ser humano no atendimento de suas necessidades básicas, ensinando o devido cuidado; a assistencial não epecífica, que é exercida juntamente com a equipe de saúde e que visa a promoção, proteção e recuperação da saúde e a reabilitação de indivíduos, famílias e comunidade; e as funções de ensino, pesquisa e administração de serviços de enfermagem;

- na prestação de assistência de enfermagem o enfoque comunitário independe do local onde as ações de saúde ou de enfermagem são executadas, isto é, pode estar presente intra e extra-muros, pois, na história natural de qualquer enfermidade, que é um processo amplo e contínuo, o hospital atende somente a uma parte. Independe, também, da presença da (o) enfermeira (o) ou de pessoal especializado em prestar assistência junto à comunidade; 
- a enfermagem comunitária é exercida, diretamente, por enfermeiras (os) com preparo e/ou experiência de assistência extra-muros, nos hospitais ou em unidades sanitárias tipo centro de saúde $e$, indiretamente, por pessoal auxiliar treinado e supervisionado pela (o) enfermeira (o). A enfermeira comunitária tem as seguintes funçôes, nos serviços de saúde comunitária: a assistência específica, que consiste em assistir ao ser humano no atendimento de suas necessidades básicas, ensinando o devido cuidado e enfocando a família e a comunidade, como unidade de planejamento e execução e avaliação dessa assistência; a assistência não específica, que compreendem atividades comuns a toda a equipe de saúde e que visam a promoção, proteção e recuperação da saúde, de indivíduos, famílias e comunidade, além de ensino, pesquisa e administração de serviços de saúde e de enfermagem;

- na função assistencial específica, a enfermeira aplica o processo de enfermagem ao nível de indivíduo, incluindo aspectos preventivos e com enfoque comunitário;

- na função assistencial especifica, a enfermeira comunitária aplica o processo de enfermagem a nível de família e comunidade, incluindo aspectos preventivos;

- o ensino de enfermagem está voltado para atender às necessidades prioritárias da comunidade e de grupos vulneráveis da população e não somente de grupos limitados, como pacientes hospitalizados que demandam espontaneamente e podem utilizar, com facilidade, os serviços de saúde existentes; procura formar pessoal para aumentar a eficiência e eficácia dos serviços que prestam assistência sanitária às populações; abarca todos os aspectos da vida humana (bio-psico-sociais) e faz com que os professores e alunos participem e se sintam responsáveis pela saúde da comunidade em que vivem; visa preparar profissionais capazes de modificar, para melhor, a situação dessa mesma comunidade e da enfermagem em geral.

\section{Marco conceitual:}

"O individuo, a família e a comunidade e suas necessidades relacionadas com o processo saúde e doença." 

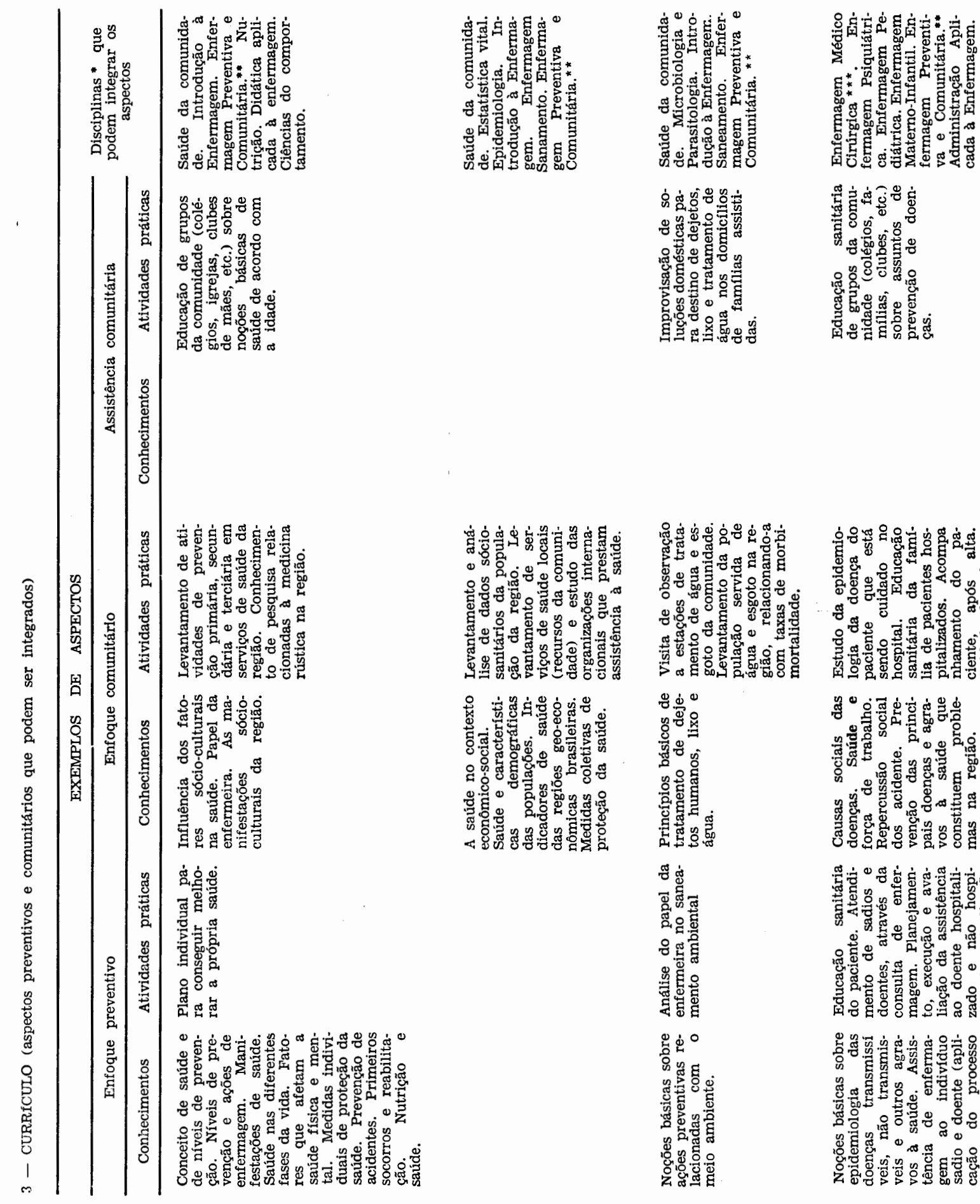

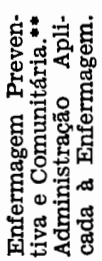
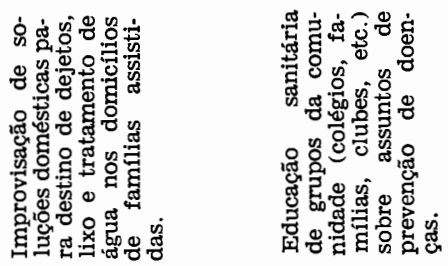

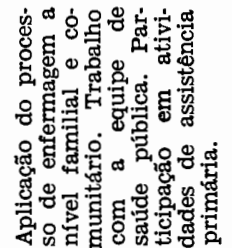

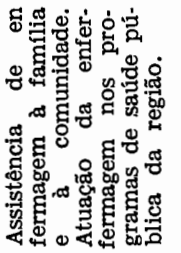

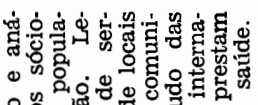

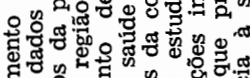

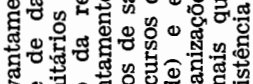

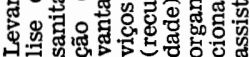

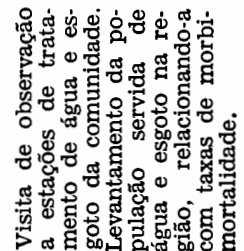

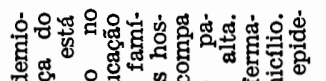

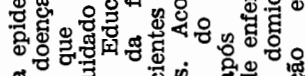
ซึ

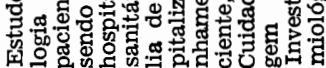
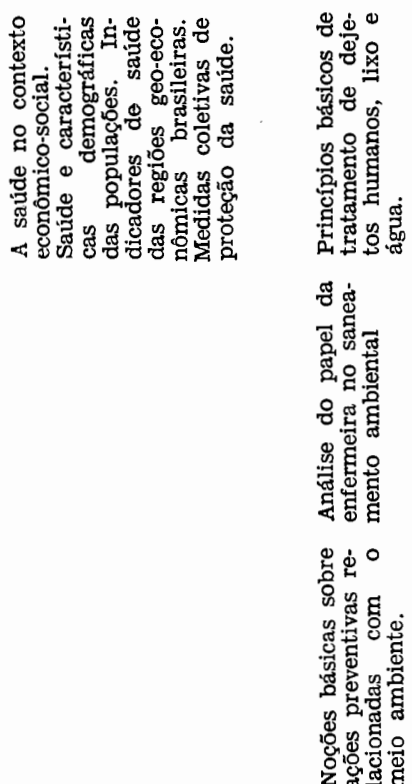

ช้ 密焉

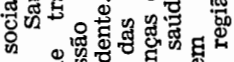

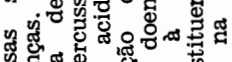

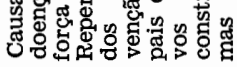

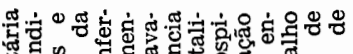

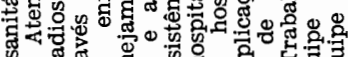
w

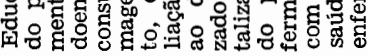

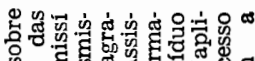

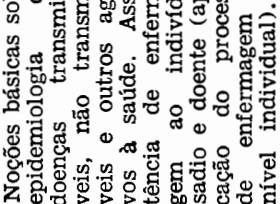


NOGUEIRA M. J. de C. The community nursing in the nursing curriculum (part II). Rev. Esc. Enf. USP, São Paulo, 13(2): 171-181, 1979.

The first part of this article contained the basic concepts on health, public health and community nursing. This part includes the problems found in community health services and presents suggestions about the functions of the public health nurse.

\section{REFERENCIAS BIBLIOGRAFICAS}

1. ADAMI, N. P. - Funções da enfermeira nos centros de saúde da Secretaria de Estado da Saúde. São Paulo, FSP/USP, 1978.

2. BRASIL, Leis e Decretos - Decreto n. 16.300 , de 31 de setembro de 1923 . In: Enfermagem: leis, decretos e portarias. Rio de Janeiro, FSESP, 1959

3. Parecer n..$^{\circ}$ 271, de 19 de outubro de 1962. Documenta, Brasília, (10): 59, dez. 1962.

4. HEIDGERKEN, L. - Quando um currículo está integrado. Rev. Bras. Ent., Rio de Janeiro, 13: 230-7, jun. 1960.

5. NOGUEIRA, M. J. de C. - Tentativa de avaliação do ensino de enfermagem de saúde pública em nível de graduação, nas escolas de enfermagem do Estado de São Paulo. São Paulo, 1972. (Tese de Doutoramento - Escola de Enfermagem da USP).

6. ORGANIZACION MUNDIAL DE LA SALUD - Fnfermería y salud de la comunidad. Ginebra, OMS, 1974. (Serie de Informes Técnicos, 558).

7. RIVERA, S. - Formas de integração do ensino. Rev. Bras. Enf., Rio de Janeiro, 13: 68.77, mar. 1960.

\section{BIBLIOGRAFIA CONSULTADA}

1. BRASIL - Ministério da Saúde/Ministério da Educação e Cultura. Relatório do seminário sobre ensino de enfermagem de saúde pública nas escolas de enfermagem do país. Rio de Janeiro, 1967. (mimeografado).

2. BRASIL - Ministério da Saúde/Ministério da Educação e Cultura. Padrões Minimos de assistência de enfermagem à comunidade. Brasília, 1977.

3. Modelo geral de enfermagem. Brasília, 1977.

4. BRASIL - Ministério da Educação e Cultura. Habilitação básica em saúde. Rio de Janeiro, FIOCRUZ, 1975.

5. NOGUEIRA, M. J. de C. et alii - Manual para o ensino de enfermagem de saúde pública. São Paulo, Sociedade Beneficente São Camilo, 1978.

6. ORGANIZACION MUNDIAL DE LA SALUD - Enseñanza de enfermería medico-quirúrgica en las escuelas de enfermería de América Latina. Washington, OPS/OMS, 1971. (Publicación Científica, 242).

7. Enseñanza de enfermeria materno-infantil en las escuelas de enfermería de America Latina. Washington, OPS/OMS, 1973. (Publicación Científica, 260).

8. Enseñanza de enfermería a nivel universitário. Washington, OPS/OMS, 1973. (Publicación Científica, 259).

9. La epidemiologia y la enfermeria. Washington, OPS/OMS, 1976. (Publicación Cientí-

fica, 333).

10. - El papel de la enfermería en la atención primaria de salud. Washington, OPS/OMS

Informe del seminario en enfermería de salud pública. Washington, OPS/OMS, 1962. (Informe sobre enfermeria, 1). 